\title{
Differences in treatment patterns among patients with castration-resistant prostate cancer treated by oncologists versus urologists in a US managed care population
}

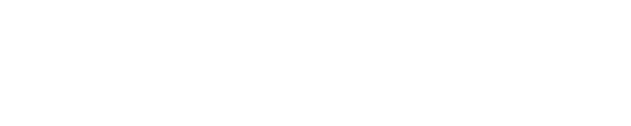

\author{
Nicole M Engel-Nitz' \\ Berhanu Alemayehu ${ }^{2}$ \\ David Parry 3 \\ Faith Nathan ${ }^{2}$ \\ 'Innovus, Eden Prairie, MN, USA; \\ ${ }^{2}$ AstraZeneca, Wilmington, DE, USA; \\ ${ }^{3}$ AstraZeneca UK, London, UK
}

Correspondence: Berhanu Alemayehu AstraZeneca, I800 Concord Pike, Wilmington, DE 19850-5437, USA

Tel + I 3028854348

Fax +l 3028855512

Email berhanu.alemayehu@astrazeneca.com
Objective: Differences in treatment patterns, health care resource utilization, and costs between patients with castration-resistant prostate cancer (CRPC) treated by oncologists and those treated by urologists were examined.

Methods: Patients aged $\geq 40$ with CRPC were identified using claims from a large US managed health care plan between July 2001 and December 2007. A 6-month baseline period was used to assess patient characteristics. Patients with visits to an urologist, without visits to an oncologist, were assigned to the urology cohort, and patients with visits to an oncologist, with or without visits to an urologist, were assigned to the oncology cohort. Treatment patterns, health care resource utilization, and costs during a variable follow-up period were compared between cohorts using descriptive statistics and Lin's regression.

Results: The urology cohort had fewer comorbid illnesses $(P<0.001)$ and patients were less likely to have other cancers during baseline $(P<0.001)$ or to die during follow-up $(P=0.004)$ compared with the oncology cohort. The oncology cohort patients were significantly more likely to have a claim for hormones $(74.5 \%$ vs $61.1 \% ; P<0.001)$, chemotherapy ( $46.9 \%$ vs $10.2 \%$, $P<0.001)$, and radiation $(22.3 \%$ vs $3.7 \%, P<0.0001)$ over follow-up. Mean unadjusted health care costs were higher in the oncology vs the urology cohort (US $\$ 31,896$ vs US $\$ 15,318$, respectively; $P<0.001)$. At 6 years follow-up, cumulative adjusted $\mathrm{CRPC}$-specific costs were significantly higher among patients treated by oncologists with chemotherapy than among patients treated by urologists.

Conclusion: CRPC patients treated by oncologists had greater use of hormones, chemotherapy, and radiation; higher percentages of patients with inpatient stays, emergency room, and ambulatory visits; and higher health care costs, than patients treated by urologists.

Keywords: prostatic neoplasms, castration, treatment protocols, oncology, urology

\section{Introduction}

Prostate cancer is the most common malignancy diagnosed among men in the US, ${ }^{1}$ with an incidence rate of 156.9 per 100,000 men. ${ }^{2}$ It has been reported that one in six American men will develop prostate cancer during the course of his lifetime..$^{3,4}$ Incidence rates vary considerably by country; by far the highest rates are seen in the US and Canada. ${ }^{5}$ In 2010, about 217,730 new cases of prostate cancer will have been diagnosed in the US, and roughly 32,050 men will have succumbed to the disease. ${ }^{2}$

Most patients with prostate cancer die from other causes and not from the disease itself, ${ }^{6,7}$ especially in cases where the cancer is slow-growing (as is the case in two-thirds 
of patients), asymptomatic, or nonmetastatic..$^{89}$ Survival rates are high for localized and regional prostate cancer (100\%), but drop dramatically to $31 \%$ for metastatic disease. ${ }^{9}$

Castration-resistant prostate cancer (CRPC) is defined by disease progression despite castrate levels of testosterone and may present as one or any combination of a continuous rise in serum levels of prostate-specific antigen, progression of pre-existing disease, or appearance of new metastases. Patients with CRPC have a poorer prognosis than patients with earlier-stage prostate cancer and frequently have metastatic disease, especially to bone. Men with CRPC who are resistant to docetaxel-based or other systemic therapy invariably progress and die from the disease..$^{10}$ According to the National Cancer Institute, metastatic prostate cancer (HRPC) was associated with a relative 5-year survival rate of $30.2 \%$ during the period 2003-2007, in contrast to 100\% 5-year survival rates among patients with localized or regional prostate cancer. ${ }^{11}$ In the IMPACT study, a Phase III study of patients with advanced prostate cancer, median survival time for patients with metastatic CRPC was 21.4 months, with slightly improved survival (25.9 months) among men receiving the Provenge ${ }^{\circledR}$ (sipuleucel-T, Dendreon, Seattle, WA) vaccine. ${ }^{12}$

Treatment options for CRPC include secondary hormonal manipulations, palliative radiotherapy, and radiotherapeutics (eg, strontium-89), steroids, bisphosphonates to preserve bone health and to prevent skeletal complications in patients with bone metastases, and the chemotherapeutic agents docetaxel, estramustine, mitoxantrone, and cabazitaxel. ${ }^{13-17}$ Although treatments for advanced prostate cancer have largely focused on palliative care, more men are likely to be offered chemotherapy for advanced-stage disease than in the past because of the survival benefit reported with docetaxel-based chemotherapy across patient subgroups. ${ }^{18}$ Based on the significant survival benefit conferred by docetaxel in patients with metastatic hormone-resistant prostate cancer (HRPC) and CRPC ${ }^{16,19}$ and its demonstrated effectiveness across a wide range of ages, ${ }^{20,21}$ docetaxel-based chemotherapy has become the current standard of care for patients with CRPC. However, it is not considered curative and, in some clinical trials, has been shown to increase the median survival by only 2.4 months. ${ }^{16}$ In June 2010, the FDA approved Jevtana ${ }^{\circledR}$ (cabazitaxel, sanofi-aventis, Bridgewater, NJ) injection in combination with prednisone for the treatment of patients with metastatic HRPC previously treated with a docetaxel-containing treatment regimen. In addition, Provenge, an autologous cellular immunotherapy, was approved by the FDA in April 2010 for treatment of asymptomatic or minimally symptomatic metastatic CRPC after a survival advantage was demonstrated.

The care of men with advanced prostate cancer is generally under the direction of urologists, although multispecialty groups of urologists and radiation oncologists working in tandem have become increasingly common. ${ }^{22}$ A study in which 3000 urologists were surveyed showed that only $4 \%$ of the 654 urologists who responded were trained to administer chemotherapy to the $64 \%$ of their patients who had prostate cancer, and roughly $30 \%$ of their patients were referred to an oncologist. However, only $19 \%$ of the patients under the care of these urologists had HRPC and 21\% had metastatic disease. $^{23}$

Investigators have called for an integrated multidisciplinary approach to treatment involving urologists, oncologists, radiation oncologists, and pathologists to ensure optimal patient care. ${ }^{24-27}$ Patients benefit from such an approach because of the spectrum of expertise encompassed by these various specialists, with the potential for more comprehensive health care if treatment is sought from more than one type of specialist. Previous studies have compared prostate cancer treatment approaches of urologists to those of oncologists, ${ }^{23,28-31}$ but they have largely been confined to the study of patients with early-stage disease, leaving a gap in understanding of the comparative treatment approaches between these two types of specialists for latestage disease.

Because of the prolonged survival and intense treatment needs CRPC patients can have after diagnosis, there is an increased likelihood that their use of health care resources and costs could represent a significant economic burden. ${ }^{13,32}$ In general, prostate cancer is associated with expenditures of roughly US\$7 billion annually ${ }^{33,34}$ and was reported to be the third most costly cancer among elderly Medicare patients diagnosed in 2004. ${ }^{35}$ There is a greater preponderance of literature on the economic burden of prostate cancer in a general population of US patients rather than the CRPC population.

The present study was designed to assess treatment patterns, health care resource utilization, and costs in a population of patients identified with CRPC, stratified by type of specialist (oncologist or urologist) visited by the patient.

\section{Methods \\ Study design}

This was a retrospective claims data analysis using medical and pharmacy data, enrollment information, and laboratory results obtained from a large US managed care claims 
database. Data from July 1, 2001 through December 31, 2008 (the study period) were used. As of 2007, data for approximately 35.4 million individuals receiving both medical and pharmacy benefits were available.

\section{Patient identification}

Patients were identified from a previously conducted burdenof-illness study. ${ }^{20}$ To summarize patient selection for that study, initial inclusion criteria required commercially insured and Medicare Advantage enrollees to be at least 40 years of age as of the year of the index date. Loosely defined, the index date was the time when patients' cancer became castration resistant. Moreover, patients had to have at least one medical claim with an International Classification of Diseases, Ninth Revision, Clinical Modification (ICD-9-CM) diagnosis code for prostate cancer (185 [malignant neoplasm of the prostate] or 233.4 [prostate cancer]), to be continuously enrolled in the health plan for 180 days prior to the index date (pre-index period) and for a minimum of 30 days following the index date (post-index period), and to have evidence of surgical or medical castration during the study period. A more detailed description of the study population selection was published previously. $^{20}$

Included patients were required to have had at least three laboratory results for the prostate-specific antigen (PSA) test between the castration date and December 1, 2007; however, hormone-treated patients with fewer than three PSA laboratory values were considered for study inclusion if they received at least one of the following services, which must have occurred at least 2 days following surgical castration or 14 days following the initiation of medical castration: a) at least one pharmacy claim or medical claim for docetaxel, b) a bone X-ray or bone scan, c) at least one PSA test, d) at least one office visit with an oncologist for prostate cancer, or e) at least one office visit.

Patients defined as having known CRPC or as not having CRPC based on PSA results were selected for inclusion in a model of CRPC status in order to determine which patients initially assigned an unknown CRPC status likely had CRPC. Logistic regression was used to model known CRPC status as a function of age, baseline comorbidity, baseline CRPCspecific costs, length of post-index enrollment, time from castration to index date, docetaxel use, evidence of bone $\mathrm{X}$-ray or bone scan, number of PSA tests identified in the medical claims, and evidence of oncology or urology office visits. A detailed explanation of this modeling approach and its application to the patient sample is provided in the previously published article by Alemayehu et al. ${ }^{20}$

\section{Cohort assignment by physician specialty}

Assignment to either the oncologist (oncology) cohort or the urologist (urology) cohort was based on the specialty of the physician visited by the patient during the study period. Patients with at least one visit to an oncologist (at any nonlaboratory site) during either the baseline or follow-up period, with or without visits to an urologist, were assigned to the oncology cohort, and patients with at least one visit to an urologist (at any nonlaboratory site) during either the baseline or follow-up period, without a visit to an oncologist, were assigned to the urology cohort. The oncology cohort includes a high percentage of patients seen by both an urologist and an oncologist. Typically, patients who are not deemed by an urologist to be candidates for chemotherapy would not be referred to an oncologist.

\section{Study measures}

Patient demographic and clinical characteristics were evaluated at baseline. Index month and year of diagnosis of CRPC, patient age, category of age (40-54; 55-64; 65-74; 75+), insurance type (commercial or Medicare Advantage), and geographic census region in which the patient was enrolled in a health plan (Northeast, South, West, or Midwest) were determined from enrollment data and medical and pharmacy claims. Clinical characteristics included pre-index Charlson comorbidity score, pre-index comorbid conditions, evidence of death during follow-up based on hospital discharge status, and evidence of other cancer based on Agency for Healthcare Research and Quality (AHRQ) Clinical Classification disease condition variables created in the original study for the pre-index period.

The use of hormone therapy, chemotherapy (see Appendix), radiation therapy, and the use of medications and other treatments for specific chemotherapy-related and cancer-related effects were examined during the baseline and follow-up periods using medical and pharmacy claims.

All-cause and CRPC-specific health care utilization were identified from medical claims for ambulatory visits, emergency department visits, and inpatient admissions during the pre- and post-index periods. Ambulatory visits included physician office visits and visits to an outpatient facility (including outpatient procedures, outpatient services, and outpatient laboratory and radiology). Any ambulatory or emergency department visit with a primary diagnosis of prostate cancer, primary diagnosis or procedure code for surgical or medical castration, primary diagnosis or procedure code for PSA tests (CPT codes 84152, 84153, or 84154; HCPCS G0103 or G9080; or ICD-9 diagnosis code 790.93), 
procedure code for radical prostatectomy (CPT codes 55810-55815, 55840-55845, 55866, or ICD-9 procedure code 60.5), or procedure code for docetaxel infusion (J9170) was considered to be CRPC-specific. Any inpatient stay with a primary diagnosis of prostate cancer was considered CRPC-specific. The monthly incidence of each type of visit was calculated, and the count of each type of visit during the post-index period was calculated on a per-patient per-month (PPPM) basis.

All-cause and CRPC-specific health care costs were calculated for overall costs, medical costs (costs from medical claims), and pharmacy costs (costs from outpatient pharmacy claims for leuprolide, triptorelin, goserelin, histrelin, or docetaxel), and were computed as the combined health plan- and patient-paid amounts identified from medical and pharmacy claims in the post-index period. Pharmacy costs were calculated for prescriptions filled at retail or mail-order pharmacies. Most chemotherapies were included as medical costs since they are administered in an office or inpatient setting. All pharmacy claims with a provider specialty of oncology or urology were included as CRPCspecific pharmacy costs. All pre- and post-index costs were calculated as costs PPPM, and were adjusted for inflation to 2008 dollars using the medical component of the Consumer Price Index. ${ }^{36}$

\section{Statistical analyses}

Descriptive analysis was performed to compare all baseline and follow-up measures across the oncology and urology cohorts. To assess similarities and differences between the cohorts, patient demographics, patient characteristics, and utilization were compared using chi-square tests for discrete data and $t$-tests for continuous data. PPPM incidences are presented for dichotomous utilization measures. All analyses were conducted using SAS (v 9.1; SAS Institute, Inc, Cary, NC) and Stata (v 10; StataCorp, College Station, TX).

Multivariate analyses of total and CRPC-specific costs adjusted for treatment cohort, age group, baseline radiation or hormone therapy use, and Charlson comorbidity score and included an interaction term for treatment cohort and chemotherapy use. Lin's weighted regression method ${ }^{37}$ was used to account for censored follow-up time as well as cost accumulation at multiple intervals. In this method, the first step models the probability of not being censored for all subjects. The second step uses a general linear regression weighted on the inversed probabilities of not being censored to model health care costs in patients who have complete follow-up information during the time period.
Results of the models are shown as estimated accumulated costs over the entire study period, starting with 1 year of costs through 6 years of costs; each cumulative cost figure was estimated with a separate model.

\section{Results \\ Study sample}

After applying all inclusion and exclusion criteria, a total of 2585 patients who visited an oncologist or urologist were retained for the study, with $61.5 \%$ in the oncology cohort $(n=1590)$ and $39.9 \%$ in the urology cohort $(n=995)$. As shown in Table 1, mean age was higher in the urology cohort than in the oncology cohort ( 75.5 vs 71.1 years, $P<0.001$ ). Patients in the oncology cohort had a higher average Charlson comorbidity score $(P<0.001)$ and a greater number of comorbid illnesses $(P<0.001)$, and were more likely to die during the follow-up period $(P=0.004)$ or to have other cancers during baseline $(P<0.001)$ compared to patients in the urology cohort. A lower percentage of the oncology cohort was enrolled in Medicare Advantage plans as compared to the urology cohort.

Table I Patient demographic and clinical characteristics at baseline

\begin{tabular}{|c|c|c|c|}
\hline Characteristic & $\begin{array}{l}\text { Oncology } \\
\text { cohort } \\
(n=1,590) \%\end{array}$ & $\begin{array}{l}\begin{array}{l}\text { Urology } \\
\text { cohort } \\
(n=995) \%\end{array} \\
\end{array}$ & $P$-value \\
\hline \multicolumn{4}{|l|}{ Age group } \\
\hline $40-54$ & 4.59 & 1.41 & $<0.001$ \\
\hline $55-64$ & 24.09 & 15.68 & \\
\hline $65-74$ & 31.45 & 23.82 & \\
\hline $75+$ & 39.87 & 59.10 & \\
\hline \multicolumn{4}{|l|}{ Insurance type } \\
\hline Commercial & 57.48 & 49.95 & $<0.001$ \\
\hline Medicare advantage & 42.52 & 50.05 & \\
\hline \multicolumn{4}{|l|}{ Health plan region } \\
\hline Northeast & 7.17 & 13.57 & $<0.001$ \\
\hline Midwest & 42.01 & 38.39 & \\
\hline South & 42.96 & 43.52 & \\
\hline West & 7.86 & 4.52 & \\
\hline $\begin{array}{r}\text { Final treatment } \\
\text { cohort counts }\end{array}$ & 61.51 & 38.49 & - \\
\hline $\begin{array}{l}\text { Evidence of death } \\
\text { during follow-up }\end{array}$ & 11.45 & 7.94 & 0.004 \\
\hline \multirow{3}{*}{$\begin{array}{l}\text { Evidence of other } \\
\text { cancers during } \\
\text { baseline period }\end{array}$} & 27.36 & 17.69 & $<0.001$ \\
\hline & & & \\
\hline & Mean [SD] & Mean [SD] & \\
\hline Age (continuous) & $71.05[9.72]$ & $75.52[9.39]$ & $<0.001$ \\
\hline $\begin{array}{l}\text { Charlson comorbidity } \\
\text { score }\end{array}$ & $4.85[2.44]$ & $3.73[2.17]$ & $<0.001$ \\
\hline $\begin{array}{l}\text { Number of AHRQ } \\
\text { comorbid conditions }\end{array}$ & $11.14[6.16]$ & $10.09[5.98]$ & $<0.001$ \\
\hline
\end{tabular}

Abbreviation: AHRQ, Agency for Healthcare Research and Quality. 


\section{Study measures}

Use of specific treatments during baseline period Higher percentages of patients in the oncology cohort used hormone treatment, chemotherapy, radiation, or other treatments for specific chemotherapy-related and cancer-related effects during the baseline period compared to the urology cohort (Figure 1A). Hormone treatment was the most commonly used treatment for both cohorts, utilized by $78.5 \%$ of the oncology cohort and $60.6 \%$ of the urology cohort $(P<0.001)$. Only $12.9 \%$ of the oncology cohort and $3.2 \%$ of the urology cohort used chemotherapy $(P<0.001)$.

\section{Use of specific treatments during follow-up period}

During follow-up, all treatments evaluated were used by significantly higher percentages of patients in the oncology cohort compared to the urology cohort (Figure 1B). The differences between cohorts were greatest for chemotherapy and radiation use, and the smallest difference was seen for hormone treatment.

Chemotherapy use increased considerably in the oncology cohort from the baseline period (12.9\% of patients) to the follow-up period ( $46.9 \%$ of patients). A higher percentage of patients in the oncology cohort used radiation during follow-up than during the baseline period $(42.7 \%$ vs $22.3 \%)$, and the same was true for the urology cohort, with $3.7 \%$ of patients receiving radiation treatment during the baseline period and 9.8\% using radiation during follow-up. Slightly fewer patients in the oncology cohort and slightly more patients in the urology cohort had hormone treatment during follow-up than during the baseline period. In both cohorts, luteinizing-hormone-releasing hormone (LHRH) agonists were the most commonly prescribed hormone (used by $65.4 \%$ of the oncology cohort and $54.6 \%$ of the urology cohort, $P<0.001$ ) (Table 2). There were high rates of treatments for other conditions (eg, anemia, neutropenia, pain) in both cohorts, particularly in the oncology cohort.

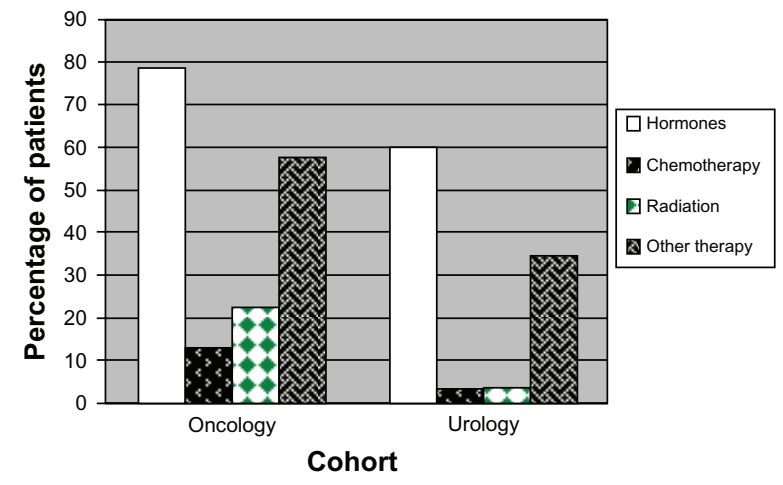

Figure IA Use of specific treatments during baseline period.

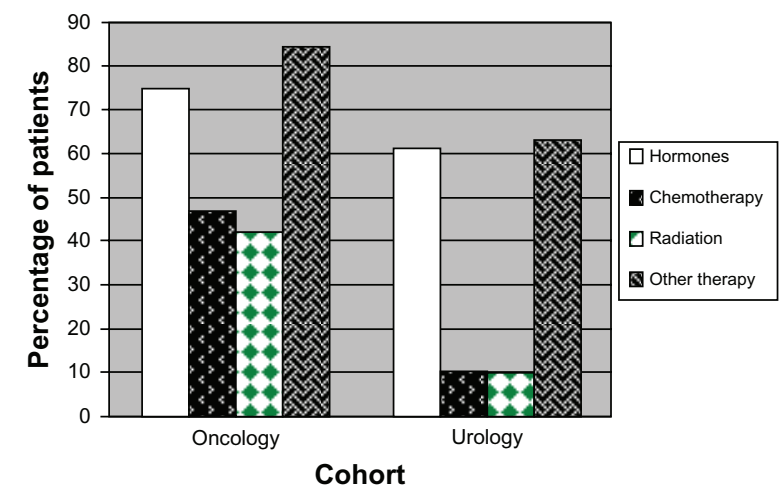

Figure IB Use of specific treatments during follow-up period.

As shown in Table 3, there were significant differences in the incidence rates of hormone treatment, chemotherapy, radiation treatment, and other medications between the cohorts ( $P<0.001$ for all comparisons).

In the oncology cohort, first use of chemotherapy after diagnosis of CRPC was highest during days 31-182 of follow-up (36.8\% of patients), whereas in the urology

Table 2 Use of specific treatments during variable-length followup period

\begin{tabular}{|c|c|c|c|}
\hline Treatments & $\begin{array}{l}\text { Oncology } \\
\text { cohort } \\
(n=1590) \%\end{array}$ & $\begin{array}{l}\text { Urology } \\
\text { cohort } \\
(n=995) \%\end{array}$ & $P$-value \\
\hline Hormone treatment & 75.4 & 61.1 & $<0.001$ \\
\hline Antiandrogens & 28.9 & 18.2 & $<0.001$ \\
\hline Aromatase inhibitors & 0.2 & 0 & \\
\hline Estrogen receptor antagonists & 0.3 & 0 & \\
\hline GnRH agonists & 6.2 & 4.7 & 0.107 \\
\hline LHRH agonists & 65.4 & 54.6 & $<0.001$ \\
\hline LHRH antagonists & 0.1 & 0 & \\
\hline Progestins & 15.7 & 6.6 & $<0.001$ \\
\hline Estrogens & 0.2 & 0.1 & \\
\hline Chemotherapy & 46.9 & 10.2 & $<0.001$ \\
\hline Alkylating & 16.5 & 3.0 & $<0.001$ \\
\hline Antimetabolites & 4.2 & 1.7 & $<0.001$ \\
\hline Docetaxel & 38.2 & 7.0 & $<0.001$ \\
\hline Other antimicrotubule & 8.9 & I.I & $<0.001$ \\
\hline Topoisomerase-active & 12.6 & 1.2 & $<0.001$ \\
\hline Antineoplastic antibiotic & 0.8 & 0.2 & \\
\hline Biologically directed & 2.8 & 0.8 & $<0.001$ \\
\hline Immune therapies & 2.7 & 0.8 & $<0.001$ \\
\hline Miscellaneous & 0.6 & 0.3 & \\
\hline $\begin{array}{l}\text { Treatments for other } \\
\text { conditions }\end{array}$ & 83.9 & 63.0 & $<0.001$ \\
\hline Anemia & 42.8 & 16.6 & $<0.001$ \\
\hline Neutropenia & 16.0 & 2.3 & $<0.001$ \\
\hline Thrombocytopenia & 2.2 & 0.3 & \\
\hline Nausea/emesis & 63.0 & 31.9 & $<0.001$ \\
\hline Pain & 67.4 & 49.9 & $<0.001$ \\
\hline
\end{tabular}

Abbreviations: $\mathrm{GnRH}$, gonadotropin-releasing hormone; LHRH, luteinizing-hormonereleasing hormone. 
Table 3 Treatment use during variable-length follow-up period - incidence rates*

\begin{tabular}{|c|c|c|c|c|c|c|c|c|}
\hline \multirow[t]{2}{*}{ Treatments } & \multicolumn{3}{|c|}{$\begin{array}{l}\text { Oncology cohort } \\
(n=1590)\end{array}$} & \multicolumn{3}{|c|}{$\begin{array}{l}\text { Urology cohort } \\
(n=995)\end{array}$} & \multicolumn{2}{|c|}{$\begin{array}{l}\text { Oncology vs } \\
\text { urology cohort }\end{array}$} \\
\hline & Events & Person-time & Rate & Events & Person-time & Rate & Ratio & $P$-value \\
\hline Hormones & 1199 & 2381 & 50.36 & 608 & 1542 & 39.42 & 1.28 & $<0.001$ \\
\hline Chemotherapy & 745 & 2381 & 31.29 & 101 & 1542 & 6.55 & 4.78 & $<0.001$ \\
\hline Radiation & 679 & 2381 & 28.52 & 97 & 1542 & 6.29 & 4.53 & $<0.001$ \\
\hline Other medications & 1334 & 2381 & 56.03 & 627 & 1542 & 40.66 & 1.38 & $<0.001$ \\
\hline
\end{tabular}

Note: *Incidence per 100 person-years.

cohort, first use of chemotherapy after diagnosis of CRPC was highest during the first 30 days of follow-up (7.0\% of patients). The mean [SD] number of days until first use of hormone therapy was 77.1 [149.7] for the oncology cohort (range, 0-1980 days) and 87.1 [141.1] for the urology cohort (range, $0-1320$ days). The mean [SD] number of days until first use of chemotherapy was 102.4 [259.0] for the oncology cohort (range, 0-2164 days) and 97.8 [223.6] for the urology cohort (range, $0-1280$ days).

\section{Health care resource utilization and cost outcomes}

\section{Baseline utilization}

Total health care utilization was higher for the oncology cohort than for the urology cohort with regard to docetaxel use during the 6-month baseline period, but differences between the cohorts were not significant for inpatient stays, emergency room (ER) visits, or ambulatory visits (Table 4).

More than $95 \%$ of each cohort had an ambulatory visit, but less than $30 \%$ of each cohort had an ER visit and roughly $20 \%$ of each cohort had an inpatient visit. Mean adjusted counts of docetaxel use, inpatient stays, and ambulatory

Table 4 Total health care utilization during baseline and followup periods

\begin{tabular}{llll}
\hline $\begin{array}{l}\text { Health care } \\
\text { utilization }\end{array}$ & $\begin{array}{l}\text { Oncology } \\
\text { cohort } \\
(\mathbf{n}=\mathbf{I 5 9 0}) \%\end{array}$ & $\begin{array}{l}\text { Urology } \\
\text { cohort } \\
(\mathbf{n}=\mathbf{9 9 5}) \%\end{array}$ & P-value \\
\hline Baseline period & & & \\
Docetaxel use & 2.64 & 0.60 & $<0.00$ I \\
Inpatient stay & 20.88 & 20.00 & 0.590 \\
ER visit & 26.86 & 23.92 & 0.097 \\
Ambulatory visit & $99.8 \mathrm{I}$ & 99.70 & 0.562 \\
Variable follow-up period & & \\
Docetaxel use & 38.24 & 7.04 & $<0.00$ I \\
Inpatient stay & 60.44 & 47.64 & $<0.00$ I \\
ER visit & 62.96 & 56.38 & $<0.00$ I \\
Ambulatory visit & 99.18 & 98.19 & $<0.00$ I \\
\hline
\end{tabular}

Abbreviation: ER, emergency room. visits were significantly higher for the oncology cohort than for the urology cohort. Length of inpatient stay did not differ significantly between cohorts; oncology patients had a mean [SD] length of stay of 8.3 [16.9] days, and urology patients had a mean [SD] length of stay of 8.4 [22.3] days. The only significant difference between cohorts was in docetaxel use, used by $2.6 \%$ of the oncology cohort and only $0.6 \%$ of the urology cohort $(P<0.001)$. However, there was a significant difference between the cohorts with regard to mean adjusted count of inpatient stays (mean [SD] 0.8 [1.2] for the oncology cohort and mean [SD] 0.6 [1.2] for the urology cohort; $P<0.001)$.

Significant differences in baseline CRPC-specific health care utilization were observed between the oncology and urology cohorts with regard to docetaxel use, inpatient stays, and ambulatory visits, but not with regard to ER visits (Table 5).

\section{Follow-up utilization}

During the variable-length follow-up period, the oncology cohort had significantly higher percentages of patients with use of docetaxel, inpatient stays, ER visits, and ambulatory visits as compared to the urology cohort (Table 4). In the

Table 5 CRPC-specific health care utilization during baseline and follow-up periods

\begin{tabular}{|c|c|c|c|}
\hline $\begin{array}{l}\text { Health care } \\
\text { utilization }\end{array}$ & $\begin{array}{l}\text { Oncology } \\
\text { cohort } \\
(n=1590) \%\end{array}$ & $\begin{array}{l}\begin{array}{l}\text { Urology } \\
\text { cohort } \\
(n=995) \%\end{array}\end{array}$ & $P$-value \\
\hline \multicolumn{4}{|l|}{ Baseline period } \\
\hline Docetaxel use & 2.64 & 0.60 & $<0.001$ \\
\hline Inpatient stay & 9.43 & 4.12 & $<0.001$ \\
\hline ER visit & 13.77 & 14.47 & 0.619 \\
\hline Ambulatory visit & 98.93 & 95.48 & $<0.001$ \\
\hline \multicolumn{4}{|c|}{ Variable follow-up period } \\
\hline Docetaxel use & 38.24 & 7.04 & $<0.001$ \\
\hline Inpatient stay & 35.72 & 12.16 & $<0.001$ \\
\hline ER visit & 33.58 & 34.87 & 0.328 \\
\hline Ambulatory visit & 97.99 & 93.07 & $<0.001$ \\
\hline
\end{tabular}

Abbreviations: CRPC, castration-resistant prostate cancer; ER, emergency room. 
oncology cohort, $63.0 \%$ had ER visits over the variablelength follow-up period (range, 30 days to 6 years), vs $56.4 \%$ of the urology cohort $(P<0.001)$. Almost all patients in both cohorts had an ambulatory visit during follow-up. More than half of the oncology cohort (60.4\%) and slightly less than half of the urology cohort (47.6\%) had an inpatient stay. There were significant differences between the cohorts with regard to mean per-6-month number of docetaxel claims, ER visits, and ambulatory visits.

Significant differences in CRPC-specific health care utilization between the oncology and urology cohorts were seen during the variable follow-up period with regard to docetaxel use, inpatient stays, and ambulatory visits, but not ER visits (Table 5).

\section{Baseline costs}

Mean total health care costs were US\$14,004 for the oncology cohort and US\$7587 for the urology cohort, $P<0.001$. Costs for ambulatory visits (office/outpatient visits) accounted for the highest mean total costs for both cohorts (Figure 2A).

The lowest cost burden for both of the cohorts was ERrelated costs. There were significant differences between the cohorts with regard to mean total, medical, ambulatory, and pharmacy costs, but inpatient costs, ER costs, and other medical costs were not significantly different between the two cohorts.

Mean baseline CRPC-specific total costs were US\$8065 for the oncology cohort and US\$3489 for the urology cohort, $P<0.001$ (Figure 3A).

Costs for ambulatory visits were the greatest expense for both cohorts, with a mean of US\$6163 and US\$2586 for the oncology and urology cohorts, respectively; the difference between the cohorts was significant $(P<0.001)$.

The category with the lowest overall cost in both cohorts was ER visits. Pharmacy costs were significantly higher for

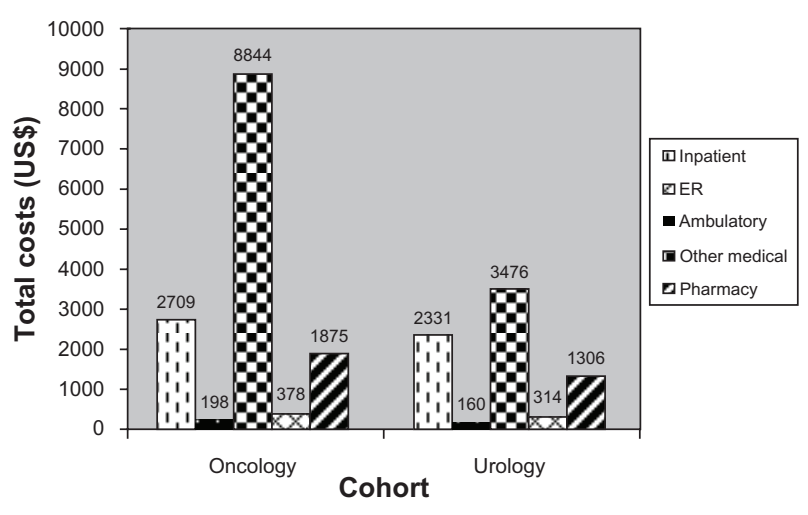

Figure 2A Baseline total health care costs (6-month), mean US\$.

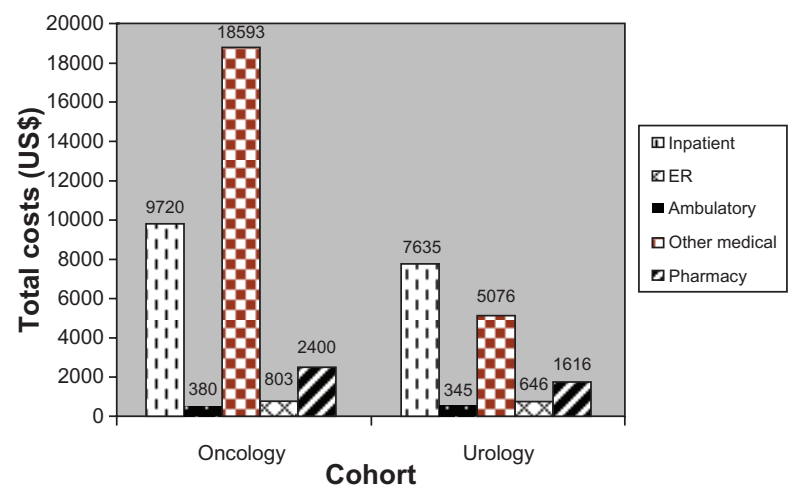

Figure 2B Follow-up total health care costs (mean, US\$).

the oncology cohort (mean US\$703) than for the urology cohort (mean US\$226), $P<0.001$. A significant difference between the cohorts was also observed for inpatient costs, but these costs were low for both cohorts (mean US\$ $\$ 870$ for the oncology cohort and US\$365 for the urology cohort, $P<0.001)$.

\section{Follow-up costs}

Total health care costs per 6 months were significantly higher for the oncology cohort than for the urology cohort during the variable-length follow-up period, with a mean of US\$31,896 per 6 months for the oncology cohort and a mean of US\$15,318 per 6 months for the urology cohort $(P<0.001)$. Ambulatory visits were the biggest cost driver for the oncology cohort (mean US\$18,593), and inpatient costs represented the highest cost burden for the urology cohort (mean US\$7635) (Figure 2B).

Mean total CRPC-specific costs per 6 months were US\$21,518 for the oncology cohort and US\$6474 for the urology cohort $(P<0.001$; Figure 3B).

Significant differences were observed between the cohorts with regard to CRPC-specific medical costs, inpatient

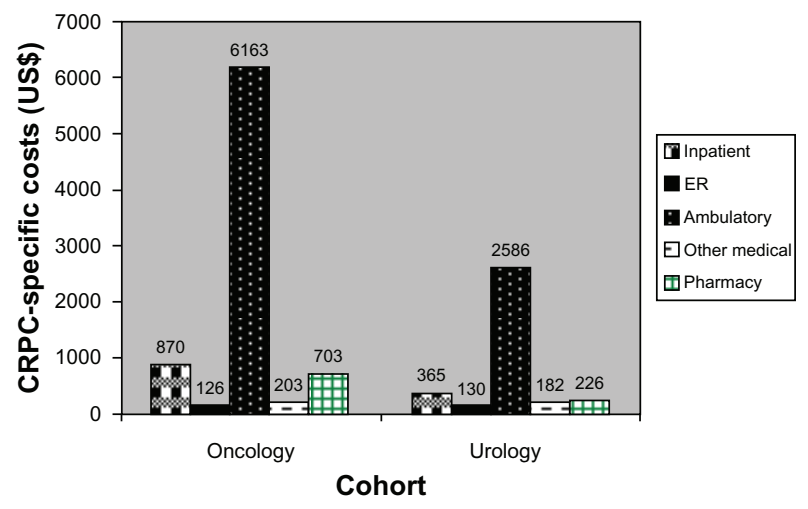

Figure 3A Baseline CRPC-specific costs (6-month), mean US\$. Abbreviation: CRPC, castration-resistant prostate cancer. 


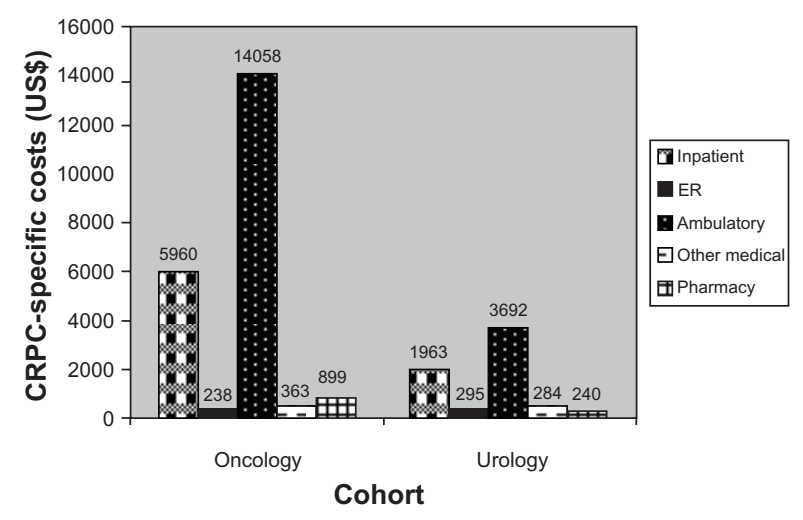

Figure 3B Follow-up CRPC-specific costs (6-month), mean US\$ Abbreviation: CRPC, castration-resistant prostate cancer.

costs, ambulatory costs, and pharmacy costs. Ambulatory visits were the biggest cost driver for both cohorts, with mean costs of US $\$ 14,058$ and US\$3682 for the oncology and urology cohorts, respectively.

The lowest costs observed in the oncology cohort were for ER visits (mean US\$238), and the lowest category of expense for the urology cohort was pharmacy costs (mean US\$240).

\section{Multivariate analyses}

Results of Lin's regression analysis confirmed the finding that the oncology and urology cohorts differed significantly with regard to total costs over the follow-up period, but only when patients were using chemotherapy. During the first year of follow-up, total adjusted health care costs were US $\$ 31,792$ (urology cohort), US\$54,306 (oncology with chemotherapy, $P<0.001$ ), and US\$30,894 (oncology without chemotherapy) (Table 6).

Patients in the oncology cohort using chemotherapy had an adjusted cumulative total cost of US $\$ 168,794$ over 6 years of follow-up; those in the oncology cohort without chemotherapy, US\$114,180; and patients in the urology cohort, $\$ 86,706$.

In addition to cohort, other factors that were statistically significantly associated with total health care costs were being in the 55-64 age group, number of PSA tests during follow-up (both of which were associated with an increase in costs), and baseline use of docetaxel (associated with a decrease in costs).

Lin's regression was also used to assess CRPC-specific costs over the follow-up period while accounting for the variable length of follow-up in the study population. Again, the urology and oncology cohorts differed significantly, but the degree of difference varied according to whether patients were using chemotherapy. Over 6 years of follow-up, cumulative adjusted CRPC-specific costs were significantly higher among patients treated by oncologists with chemotherapy than among patients treated by urologists (US\$68,286 vs US\$25,081, $P<0.001)$. However, statistically significant differences in adjusted cumulative CRPC-specific costs were not observed between patients treated by oncologists without chemotherapy (US\$32,927) and patients treated by urologists, regardless of follow-up length.

Factors other than cohort that were statistically significantly associated with CRPC-specific costs were comorbidity score (associated with a decrease in costs), being in the 55-64 age group (associated with an increase in costs), and number of PSA tests during follow-up (associated with an increase in costs).

\section{Discussion}

The primary outcomes evaluated in this study indicate that disparate treatment patterns are observed between oncologists and urologists, regardless of differences in patient characteristics and variations in disease severity.

Table 6 Predicted cumulative total and CRPC-specific health care costs (adjusted)

\begin{tabular}{|c|c|c|c|c|c|c|}
\hline \multirow[t]{2}{*}{ Cohort } & \multicolumn{6}{|c|}{ Cumulative years } \\
\hline & I & $1-2$ & $1-3$ & $1-4$ & I-5 & $1-6$ \\
\hline \multicolumn{7}{|c|}{ Adjusted total health care costs (US\$) } \\
\hline Urology cohort (ref.) & $31,791.58$ & $49,463.35$ & $64,476.63$ & $72,970.69$ & $83,089.42$ & $86,706.26$ \\
\hline Oncology with chemotherapy & $54,305.81 \ddagger$ & $92,096.22^{\dagger}$ & $123,586.28^{\ddagger}$ & $150,633.48^{\ddagger}$ & $159,824.79 \ddagger$ & $168,793.50$ \\
\hline Oncology without chemotherapy & $30,894.37$ & $54,351.70$ & $72,104.95$ & $89,200.67^{\dagger}$ & $103,629.01^{*}$ & $1 \mid 4,179.69^{\dagger}$ \\
\hline \multicolumn{7}{|c|}{ Adjusted CRPC-specific costs (US\$) } \\
\hline Urology cohort (ref.) & $10,666.83$ & $16,259.84$ & $20,028.80$ & $22,226.95$ & $23,692.21$ & $25,081.09$ \\
\hline Oncology with chemotherapy & $26,042.74^{\ddagger}$ & $41,259.20^{\ddagger}$ & $53,476.65^{\ddagger}$ & $63,464.28^{\ddagger}$ & $66,685.7 I^{\ddagger}$ & $68,286.20^{\ddagger}$ \\
\hline Oncology without chemotherapy & $9,648.83$ & $16,216.14$ & $20,655.64$ & $24,067.06$ & $29,942.43$ & $32,927.43$ \\
\hline
\end{tabular}

Notes: $* P<0.05 ;{ }^{\dagger} P<0.01$; ${ }^{\ddagger}<0.001$.

Abbreviations: CRPC, castration-resistant prostate cancer; ref, reference group. 
Patients appear to benefit from an integrated multidisciplinary approach and may receive more comprehensive health care if they seek treatment from more than one type of specialist. $^{18,24,25}$

Incidence rates of medication use, including hormones and chemotherapy, were significantly higher among patients treated by oncologists than among those treated by urologists.

During the 6-month baseline and variable follow-up periods in our study, all treatments evaluated were used by significantly higher percentages of patients in the oncology cohort as compared to the urology cohort; the largest differences between cohorts were seen for chemotherapy and radiation use. For patients in the oncology cohort, use of chemotherapy, radiation treatment, and other therapies increased considerably from the baseline to the variablelength follow-up period. The oncology cohort had significantly higher percentages of patients with use of docetaxel, inpatient stays, ER visits, and ambulatory visits compared to the urology cohort during the follow-up period. More than half of the patients in the oncology cohort and just under half of the urology cohort patients had inpatient stays during the follow-up period.

For both the oncology and urology cohorts, results of cost analyses indicate that the economic burden increases significantly as the disease progresses, both with regard to mean total health care costs and mean CRPC-specific total costs.

During the variable follow-up period, cost differences between the cohorts were significant for overall and CRPCspecific total costs, medical costs, ambulatory costs, and pharmacy costs, as well as for CRPC-specific inpatient costs (but not for overall inpatient costs). After adjusting for other factors, adjusted total and CRPC-specific costs did not significantly differ between the cohorts when the use of chemotherapy among oncologists' patients was factored into the analysis. During the first year of follow-up, total adjusted health care costs for the urology cohort were very similar to those seen for the oncology cohort without chemotherapy, but during years $1-6$, adjusted total costs were much higher for the oncology cohort without chemotherapy than for patients in the urology cohort.

The costs observed in our study are in line with those reported in previous studies. A retrospective study of 3000 prostate cancer patients receiving androgen deprivation therapy reported a mean total cost of health care of US\$48,350 per patient over 36 months. ${ }^{38}$ In a study of 4553 patients with prostate cancer identified from a national registry, mean annual prostate cancer-related costs were US\$7740 per patient, but the average cost per patient varied considerably by treatment type. ${ }^{39}$ And in a retrospective study of 2056 patients with metastatic prostate cancer, average annual cost was US\$30,626 per patient, as compared to an average annual cost of US\$18,948 for those patients with PSA progression. ${ }^{40}$

In our study, the first year of adjusted total costs comprised $36 \%$ of the total costs at the end of the follow-up period for the oncology cohort and $27 \%$ of the total costs for the urology cohort. Evaluating only CRPC-specific costs, the first year of costs comprised $29 \%$ of the 6-year costs for the oncology without chemotherapy cohort, $38 \%$ of the 6-year costs for the oncology with chemotherapy cohort, and $43 \%$ of the final costs for the urology cohort. These percentages are somewhat lower than those reported by Skolarus et al, ${ }^{33}$ who identified 105,961 patients diagnosed with prostate cancer between 1992 and 2005 using SEER-Medicare data and found that, during the entire study period, the initial phase (ie, first year) of prostate cancer care comprised the greatest financial burden at US\$987,774,979 (58\% of total costs), followed by continuing care (32\%) and end-of-life care $(10 \%)$. However, the Skolarus et $\mathrm{a}^{33}$ estimates pertained to the initial phase of prostate cancer care, not the initial phase of CRPC care; patients with CRPC likely received treatment for their prostate cancer prior to their diagnosis of CRPC and those costs are not captured in the follow-up costs for the study population.

CRPC-specific mean total health care costs comprised $67 \%$ of overall mean total health care costs in the oncology cohort, and $42 \%$ in the urology cohort. These findings are in line with results reported by Fitzpatrick et al, ${ }^{18}$ who reported that average prostate cancer-related costs made up about $50 \%$ of the total all-cause health care costs incurred by their patients, and by Crawford et al, ${ }^{41}$ who found that prostate cancer-related costs represented $60 \%$ of the average total expenditures for the 2-year period after initial diagnosis. Krahn et $\mathrm{al}^{42}$ found a higher proportion of total costs to be attributable to prostate cancer in the first year after diagnosis $(72 \%)$ but prostate cancer-specific costs comprised only $18 \%-35 \%$ of total costs in the other phases of their study.

This study was designed to examine differences in treatment patterns and differences in total and CRPC-specific health care costs and resource utilization between patients with CRPC treated by oncologists and those treated by urologists in a selected sample of 2585 patients. Our aim was 
not to determine which patients fared better or to compare the quality of care delivered by these two types of specialists. Attempting to make such a comparison would not be feasible given the differences in patient characteristics between the two cohorts; namely, patients in the oncology cohort had greater comorbidity and were more likely to die during the follow-up period or to have other cancers during baseline as compared to patients in the urology cohort.

\section{Study limitations}

Inherent limitations of retrospective claims analyses, as well as limitations specific to this study, should be considered when interpreting these results. There are limits to the degree to which claims data can accurately capture an individual's medical history. Claims data are collected for the purpose of payment and not research, and are subject to possible coding errors, coding for the purpose of rule-out rather than actual disease, and undercoding. Furthermore, selection of study populations from claims data is a nonvalidated methodology. Nevertheless, administrative data allow for the examination of health care utilization and expenditure patterns in a realworld setting, away from the highly controlled environment of clinical trials, and offer the advantage of large sample sizes with diverse medical histories. The presence of a prescription claim does not necessarily mean the drug was taken as prescribed, and patients who may have received drugs without the presence of a prescription claim (eg, by receiving samples from their physicians or obtaining medications outside of the health care pharmacy system) may not have been captured.

Limitations specific to this study must also be taken into account. The use of PSA levels as an indicator of CRPC, although supported by previous research, may have led to overidentification of patients with CRPC. Our results may also have been biased by our method of recording outcome measures on a PPPM basis to adjust for varying lengths of follow-up time. Another possible confounding factor is that patients who visited urologists and oncologists in the same office could not be discerned from the claims data. Furthermore, not all patients in the oncology cohort were treated exclusively by oncologists; most (83\%) were also treated by urologists. By definition, none of the patients in the urology cohort also visited an oncologist. Thus, it is difficult to determine whether differences in health care resource utilization and cost outcomes are due to a difference in treatment patterns between the two types of specialists or to other factors such as age, comorbidity, and severity of disease. As a sensitivity analysis, we examined the treatment patterns stratifying the oncologist cohort by whether the patients had also visited an urologist. Our results (tables not shown) indicated that there was a statistically significant difference in the number of comorbid conditions, with patients in the oncology-only group having an average of one fewer condition than the oncology + urology group $(P<0.001)$. However, there were no statistically significant differences between the groups with regard to demographics; Charlson comorbidity score; incidence rate of use of hormone, chemotherapy, radiation, or other therapy, or in use of specific types of chemotherapy. ${ }^{43}$

This study might have been enhanced by an examination of the costs of any previous or ongoing surgical interventions incurred by the two patient cohorts. However, data obtained for this study were limited to a 6-month pre-index period. The study patients may in fact have been undergoing treatment for several years.

It should also be noted that results may not be generalizable to the entire US prostate cancer population, such as younger patients, those with fewer comorbidities, or those with less advanced disease. And since both commercially insured and Medicare Advantage plan members were included in this study, differences in the way services are covered may have resulted in different utilization patterns among these two types of enrollees. However, the plans used for analysis encompass a wide US geographic distribution and therefore provide the capability for generalization to managed care populations on a national level.

\section{Conclusion}

This study adds to the scant literature examining costs associated with treating advanced-stage prostate cancer. A few studies have been conducted in clinical settings, but for the most part they have evaluated patients receiving a specific treatment. A clearer understanding of treatment patterns and costs associated with advanced-stage prostate cancer may help to inform treatment decisions or identify areas where new treatment options could reduce the economic burden on the health care system. Future studies evaluating the differences in practice patterns between urologists and oncologists that also assess the implications of these differing patterns in terms of outcomes would represent an important addition to the literature.

In this study, patients who were treated by oncologists, particularly those using chemotherapy, had higher total and prostate cancer-related health care costs than CRPC patients treated by urologists. In addition, CRPC patients treated by 
oncologists had greater use of hormones, chemotherapy, and radiation, and higher percentages of patients with inpatient stays, ER, and ambulatory visits, as compared to patients treated by urologists. However, oncologist-treated patients who were not using chemotherapy had treatment patterns and costs that were very similar to those observed in the urologist-treated cohort, suggesting that differences in costs between the two cohorts may be driven primarily by differences in severity of illness.

Our study appears to be the most extensive analysis of comparisons of treatment patterns, health care resource utilization, and costs between patients treated by oncologists and those treated by urologists. Previous research comparing prostate cancer treatment approaches of urologists to those of oncologists has primarily focused on patients with early-stage disease whereas we have evaluated comparative treatment approaches between these two types of specialists for late-stage disease.

\section{Acknowledgments}

The authors would like to thank Victoria Porter, a medical writer at Innovus, April Teitelbaum, MD, Senior Medical Director at i3 Research, and Mary Dominiecki, PhD, Clinical Publications Lead at AstraZeneca, for editorial assistance in the preparation of this manuscript; Mahesh Kulakodlu, a research analyst at Innovus, for specification and verification of the analytic dataset and statistical analyses; Priyanka Koka, a statistical programmer at Innovus, for programming of the analytic dataset.

\section{Disclosure}

This study was funded by AstraZeneca. The data used in the study were obtained from a large managed care database proprietary to Innovus, the company hired to conduct this study. Berhanu Alemayehu, Faith Nathan, and David Parry are employed by AstraZeneca, and Nicole Engel-Nitz is employed by Innovus. Faith Nathan, Berhanu Alemayehu, and David Parry own shares of stock in AstraZeneca.

\section{References}

1. Max W, Rice DP, Sung HY, Michel M, Breuer W, Zhang X. The economic burden of prostate cancer, California, 1998. Cancer. 2002;94(11): 2906-2913.

2. National Cancer Institute. SEER stat fact sheets: prostate. Available at: http://www.seer.cancer.gov/statfacts/html/prost.html. Accessed May 18, 2011.

3. Jemal A, Siegel R, Xu J, Ward E. Cancer statistics, 2010. Ca Cancer J Clin. 2010;60(5):277-300.

4. Altekruse SF, Kosary CL, Krapcho M, et al, editors. SEER cancer statistics review, 1975-2007, National Cancer Institute. Bethesda MD. Available at: http://seer.cancer.gov/csr/1975_2007/.Accessed May 18, 2011.
5. Quinn M, Babb P. Patterns and trends in prostate cancer incidence, survival, prevalence and mortality. Part I: international comparisons. BJU Int. 2002;90(2):162-173.

6. Chodak G. Prostate cancer: epidemiology, screening, and biomarkers Rev Urol. 2006;8(Suppl 2):S3-S8.

7. Johansson JE, Holmberg L, Johansson S, Bergstrom R, Adami HO. Fifteen-year survival in prostate cancer: a prospective, population-based study in Sweden. JAMA. 1997;277(6):467-471.

8. American Society of Clinical Oncology. Prostate cancer. Cancer. Net. Available at: http://www.cancer.net/patient/Cancer+Types/ Prostate+Cancer. Accessed May 18, 2011.

9. American Cancer Society. What is prostate cancer? Information and resources for cancer: breast, colon, prostate, lung and other forms. Available at: http://www.cancer.org/Cancer/ProstateCancer/ DetailedGuide/prostate-cancer-key-statistics. Accessed May 18, 2011.

10. Seruga B, Ocana A, Tannock IF. Drug resistance in metastatic castrationresistant prostate cancer. Nat Rev Clin Oncol. 2011;8(1):12-23.

11. National Cancer Institute. SEER relative survival rates by survival time. By Cancer Site, prostate, all ages, all races, male,1988-2004. Available at: http://seer.cancer.gov/faststats/selections.php\#Output. Accessed May 18, 2011.

12. PSA Rising. Provenge vaccine "significantly improves" survival in men with advanced prostate cancer. [Press release. February 17, 2005]. Available at: http://www.psa-rising.com/med/immun/provenge-05.htm. Accessed May 18, 2011.

13. National Comprehensive Cancer Network [NCCN]. Prostate cancer. NCCN clinical practice guidelines in oncology, v.3.2010. Fort Washington, PA. Available at: http://www.nccn.org/professionals/ physician_gls/PDF/prostate.pdf. Accessed May 18, 2011.

14. James ND, Caty A, Borre M, et al. Safety and efficacy of the specific endothelin A receptor antagonist ZD4054 in patients with hormone resistant prostate cancer and bone metastases who were pain free or mildly symptomatic: a double-blind, placebo-controlled, randomised, phase 2 trial. Eur Urol. 2009;55(5):1112-1123.

15. Saad F, Karakiewicz P, Perrotte P. The role of bisphosphonates in hormone-refractory prostate cancer. World J Urol. 2005;23(1):14-18.

16. Tannock IF, de Wit R, Berry WR, et al. Docetaxel plus prednisone or mitoxantrone plus prednisone for advanced prostate cancer. $N$ Engl J Med. 2004;351(15):1502-1512.

17. Quilty PM, Kirk D, Bolger JJ, et al. A comparison of the palliative effects of strontium-89 and external beam radiotherapy in metastatic prostate cancer. Radiother Oncol. 1994;31(1):33-40.

18. Fitzpatrick JM, Anderson J, Sternberg CN, et al. Optimizing treatment for men with advanced prostate cancer: expert recommendations and the multidisciplinary approach. Crit Rev Oncol Hematol. 2008; 68(Suppl 1):S9-S22.

19. Petrylak DP, Tangen CM, Hussain MH, et al. Docetaxel and estramustine compared with mitoxantrone and prednisone for advanced refractory prostate cancer. $N$ Engl J Med. 2004;351(15):1513-1520.

20. Alemayehu B, Buysman E, Parry D, Becker L, Nathan F. Economic burden and healthcare utilization associated with castration-resistant prostate cancer in a commercial and Medicare Advantage US patient population. J Med Econ. 2010;13(2):351-361.

21. Beer TM, Berry W, Wersinger EM, et al. Weekly docetaxel in elderly patients with prostate cancer: efficacy and toxicity in patients at least 70 year of age compared with patients younger than 70 years. Clin Prostate Cancer. 2003;2(3):167-172.

22. Schilling PJ. Formation of combined urology and radiation oncology practices: objective data from radiation oncologists for rationale. Am J Clin Oncol. August 27, 2010. [Epub ahead of print]

23. Crawford ED. The role of the urologist in treating patients with hormone-refractory prostate cancer. Rev Urol. 2003;5(Suppl 2): S48-S52.

24. Montagut C, Albanell J, Bellmunt J. Prostate cancer. Multidisciplinary approach: a key to success. Crit Rev Oncol Hematol. 2008; 68(Suppl 1):S32-S36. 
25. Jang TL, Bekelman JE, Liu Y, et al. Physician visits prior to treatment for clinically localized prostate cancer. Arch Intern Med. 2010;170(5): $440-450$.

26. Taneja SS. A multidisciplinary approach to the management of hormonerefractory prostate cancer. Rev Urol. 2003;5(Suppl 2):S53-S59.

27. Bracarda S, Logothetis C, Sternberg CN, et al. Current and emerging treatment modalities for metastatic castration-resistant prostate cancer. BJU Int. 2011;107(Suppl 2):13-20.

28. Spencer BA, Miller DC, Litwin MS, et al. Variations in quality of care for men with early-stage prostate cancer. J Clin Oncol. 2008;26(22): 3735-3742.

29. Cooperberg MR. Specialist visits (urologist, radiation oncologist, medical oncologist) are strongly associated with treatment received for prostate cancer in the USA. Evid Based Med. 2010;15(3):95-96.

30. Miller DC, Spencer BA, Ritchey J, et al. Treatment choice and quality of care for men with localized prostate cancer. Med Care. 2007;45(5): 401-409.

31. North West Uro-Oncology Group. A preliminary report on a patientpreference study to compare treatment options in early prostate cancer. BJU Int. 2002;90(3):253-256.

32. Grover SA, Zowall H, Coupal L, Krahn MD. Prostate cancer: 12. The economic burden. CMAJ. 1999;160(5):685-690.

33. Skolarus TA, Zhang Y, Miller DC, Wei JT, Hollenbeck BK. The economic burden of prostate cancer survivorship care. J Urol. 2010;184(2):532-538.

34. Roehrig C, Miller G, Lake C, et al. National health spending by medical condition,1996-2005. Health Aff (Millwood). 2009;28(2):w358.

35. Yabroff KR, Lamont EB, Mariotto A, et al. Cost of care for elderly cancer patients in the United States. J Natl Cancer Inst. 2008;100(9): 630-641.
36. US Department of Labor, Bureau of Labor Statistics. Consumer Price Index. Chained Consumer [Price Index for all urban consumers (C-CPI-U) 1999-2008], Medical Care. Series ID: SUUR0000SAM. Available at: http://data.bls.gov/cgi-bin/surveymost?su. Accessed May 18, 2011.

37. Lin DY. Linear regression analysis of censored medical costs. Biostatistics. 2000;1(1):35-47.

38. Krupski TL, Foley KA, Baser O, et al. Healthcare cost associated with prostate cancer, androgen deprivation therapy and bone complications. J Urol. 2007;178(4 Pt 1):1423-1428.

39. Wilson LS, Tesoro R, Elkin EP, et al. Cumulative cost pattern comparison of prostate cancer treatments. Cancer. 2007;109(3): 518-527.

40. Penson DF, Moul JW, Evans CP, et al. The economic burden of metastatic and prostate specific antigen progression in patients with prostate cancer: findings from a retrospective analysis of health plan data. J Urol. 2004; 171(6 Pt 1):2250-2254.

41. Crawford ED, Black L, Eaddy M, Kruepp EJ. A retrospective analysis illustrating the substantial clinical and economic burden of prostate cancer. Prostate Cancer Prostatic Dis. 2010;13(2): $162-167$.

42. Krahn MD, Zagorski B, Laporte A, et al. Healthcare costs associated with prostate cancer: estimates from a population-based study. BJU Int. 2010;105(3):338-346.

43. Engel-Nitz NM, Alemayehu B, Nathan F, Parry D. Variations in chemotherapy treatment by oncologists and urologists for patients with castration-resistant prostate cancer (CRPC). Poster presented at the Academy of Managed Care Pharmacy (AMCP) Annual Meeting, St Louis, MO, October 13-15, 2010. 


\section{Appendix}

Appendix Chemotherapy agents included in medication subclasses

\begin{tabular}{|c|c|}
\hline Subclass & Medication \\
\hline \multirow[t]{20}{*}{ Alkylating agents } & Bendamustine \\
\hline & Busulfan \\
\hline & Carmustine \\
\hline & Carmustine/polifeprosan \\
\hline & Chlorambucil \\
\hline & Cyclophosphamide \\
\hline & Dacarbazine \\
\hline & Estramustine \\
\hline & Ifosfamide \\
\hline & Lomustine \\
\hline & Mechlorethamine \\
\hline & Melphalan \\
\hline & Procarbazine \\
\hline & Streptozocin \\
\hline & Temozolomide \\
\hline & Thiotepa \\
\hline & Uracil mustard \\
\hline & Carboplatin \\
\hline & Cisplatin \\
\hline & Oxaliplatin \\
\hline \multirow[t]{16}{*}{ Antimetabolites } & Methotrexate \\
\hline & Pemetrexed \\
\hline & Cladribine \\
\hline & Clofarabine \\
\hline & Cytarabine \\
\hline & Fludarabine \\
\hline & Mercaptopurine \\
\hline & Nelarabine \\
\hline & Pentostatin \\
\hline & Thioguanine \\
\hline & Azacitidine \\
\hline & Capecitabine \\
\hline & Floxuridine \\
\hline & Fluorouracil \\
\hline & Gemcitabine \\
\hline & Hydroxyurea \\
\hline Docetaxel (taxotere) & Docetaxel \\
\hline \multirow[t]{6}{*}{ Other antimicrotubule agents } & Paclitaxel \\
\hline & Albumin-bound paclitaxel \\
\hline & Vinblastine \\
\hline & Vincristine \\
\hline & Vinorelbine \\
\hline & Ixabepilone \\
\hline \multirow[t]{4}{*}{ Topoisomerase-active agents } & Irinotecan \\
\hline & Topotecan \\
\hline & Daunorubicin \\
\hline & Doxorubicin \\
\hline
\end{tabular}

(Continued)
Appendix (Continued)

\begin{tabular}{|c|c|}
\hline Subclass & Medication \\
\hline & Pegylated liposomal \\
\hline & Doxorubicin \\
\hline & Epirubicin \\
\hline & Idarubicin \\
\hline & Mitoxantrone \\
\hline & Valrubicin \\
\hline & Etoposide \\
\hline & Teniposide \\
\hline \multirow[t]{4}{*}{ Antineoplastic antibiotics } & Bleomycin \\
\hline & Dactinomycin \\
\hline & Mitomycin C \\
\hline & Plicamycin \\
\hline \multirow[t]{22}{*}{ Biologically directed therapies } & Alitretinoin \\
\hline & Bexarotene \\
\hline & Bortezomib \\
\hline & Dasatinib \\
\hline & Erlotinib \\
\hline & Gefitinib \\
\hline & Imatinib \\
\hline & Lapatinib \\
\hline & Nilotinib \\
\hline & Sorafenib \\
\hline & Sunitinib \\
\hline & Temsirolimus \\
\hline & Tretinoin \\
\hline & Alemtuzumab \\
\hline & Bevacizumab \\
\hline & Cetuximab \\
\hline & Gemtuzumab \\
\hline & Ibritumomab \\
\hline & Panitumumab \\
\hline & Rituximab \\
\hline & Tositumomab \\
\hline & Trastuzumab \\
\hline \multirow[t]{5}{*}{ Immune therapies } & Aldesleukin (IL-2) \\
\hline & Interferon alfa-2a \\
\hline & Interferon alfa- $2 b$ \\
\hline & Lenalidomide \\
\hline & Thalidomide \\
\hline \multirow[t]{12}{*}{ Miscellaneous agents } & Altretamine \\
\hline & Arsenic trioxide \\
\hline & Asparaginase \\
\hline & Bcg vaccine \\
\hline & Decitabine \\
\hline & Denileukin \\
\hline & Levamisole \\
\hline & Mitotane \\
\hline & Pegaspargase \\
\hline & Porfimer \\
\hline & Strontium-89 \\
\hline & Vorinostat \\
\hline Zoledronic acid (zometa) & Zoledronic acid \\
\hline
\end{tabular}

\section{Dovepress}

\section{Publish your work in this journal}

Cancer Management and Research is an international, peer-reviewed open access journal focusing on cancer research and the optimal use of preventative and integrated treatment interventions to achieve improved outcomes, enhanced survival and quality of life for the cancer patient The journal welcomes original research, clinical \& epidemiological

studies, reviews \& evaluations, guidelines, expert opinion \& commentary, case reports \& extended reports. The manuscript management system is completely online and includes a very quick and fair peerreview system, which is all easy to use. Visit http://www.dovepress.com/ testimonials.php to read real quotes from published authors. 